\title{
Intensity and polarization self-pulsations in VCSELs
}

\author{
Alessandro Scirè, Josep Mulet, Claudio R. Mirasso* and Maxi San Miguel \\ Instituto Mediterráneo de Estudios Avanzados, CSIC-UIB, Campus UIB, E-07071 Palma \\ de Mallorca, Spain.
}

\begin{abstract}
We implement a dynamical model that describes the polarization behavior in VCSELs containing an absorbing region surrounding the active zone. We find four regions of qualitative different behaviors: stable linearly-polarized operation, intensity pulsations of a linearly-polarized component, pulsations of both total intensity and polarization components, and polarization self-pulsations with constant total intensity. We characterize the four regions by computing the polarization resolved optical and power spectra. The predicted scenario agrees with recent experimental results.
\end{abstract}

OCIS codes: $250.7260,260.5430$ 
Edge-emitting lasers (EELs) with a narrow-stripe geometry may exhibit self-pulsations (SPs) due to the saturable absorption region surrounding the active zone ${ }^{1}$. This phenomenon has been implemented in clock extraction systems ${ }^{2}$, in synchronization schemes for encoded communication $^{3}$ and as a method to reduce the noise of laser diodes in optical disk systems ${ }^{4}$. Vertical-Cavity Surface-Emitting Lasers (VCSELs) are important compact light sources ${ }^{5}$ for applications, e.g. in optical interconnects and optical data storage, due to their low threshold current, single-longitudinal mode operation and easy integrability in 2-D arrays. As in EELs, VCSELs may contain an unpumped region surrounding the central gain zone and therefore SPs conditions would be expected. Recently, SPs have been experimentally demonstrated in small active region VCSELs ${ }^{6,7}$. Since the polarization in VCSELs is not so well stabilized ${ }^{9}$ as in EELs, the polarization properties of self-pulsating VCSELs is an interesting issue to be investigated. In this letter, we implement a model suitable to describe SPs in VCSELs operating in the fundamental transverse mode, within the framework of the Spin-Flip Model ${ }^{9,10}$ (SFM). The absorber region is modeled as an additional carrier reservoir as is done in Yamada's model ${ }^{1}$. The complete set of equations reads

$$
\begin{aligned}
\dot{F}_{ \pm} & =\frac{1}{2}(1+i \alpha)\left[D_{1}+D_{2} \pm d_{1} \pm d_{2}-1\right] F_{ \pm}-\left(\varepsilon_{a}+i \varepsilon_{p}\right) F_{\mp}, \\
\dot{D}_{1,2} & =\gamma_{1,2}\left[\mu_{1,2}-D_{1,2}-a_{1,2}\left(D_{1,2}+d_{1,2}\right)\left|F_{+}\right|^{2}-a_{1,2}\left(D_{1,2}-d_{1,2}\right)\left|F_{-}\right|^{2}+c_{12,21} D_{2,1}\right], \\
\dot{d}_{1,2} & =-\gamma_{s 1,2} d_{1,2}-\gamma_{1,2}\left[a_{1,2}\left(D_{1,2}+d_{1,2}\right)\left|F_{+}\right|^{2}-a_{1,2}\left(D_{1,2}-d_{1,2}\right)\left|F_{-}\right|^{2}-c_{12,21} d_{2,1}\right],
\end{aligned}
$$

where the subindexes 1,2 stand for the pumped and absorbing region, respectively. $F_{ \pm}$are the slowly-varying complex amplitudes of the two circularly-polarized components of the electric field. $D_{1,2}$ are the total carrier inversion between the conduction and valence bands referred to the transparency carrier density, while $d_{1,2}$ are the differences of the carrier inversion with opposite spin orientations. The equations are written in a dimensionless form $^{8}$ such that the time is measured in $\kappa^{-1}$ units, $\kappa$ being the cavity decay rate. The effective injection currents, with respect to transparency, are $\mu_{1,2}$. Since the current is only supplied in the central region, $\mu_{1}>0$ while $\mu_{2}<0$. Carrier diffusion is introduced in the rate equations through the coupling terms $c_{12} D_{2}$ and $c_{21} D_{1}$. The linewidth enhancement 
factor $\alpha$ describes the phase-amplitude coupling in semiconductor lasers. The parameters $\gamma_{1,2}$ stand for the total carrier decay rates, while $\gamma_{s 1,2}$ are the effective spin flip rates. The phase and amplitude anisotropies ${ }^{9}$ are $\varepsilon_{p}$ and $\varepsilon_{a}$ respectively. The differential gain in the active and in the absorber regions differ due to their different carrier densities. This effect is taken into account through the parameters $a_{j}$. By a proper rescaling of the variables we can assume that $a_{1}=1$, while $a_{2}$ represents the ratio of the effective gain coefficient in the absorbing region with respect to the pumped zone. The effective gain coefficient is defined ${ }^{1}$ as the product of the differential gain times the fraction of optical power within each region.

We start our discussion looking at the linearly-polarized (LP) steady state solutions of Eqs. (1)-(3). The electrical fields are expressed as $F_{ \pm}(t)=Q e^{i\left(\omega_{ \pm} t \pm \varphi\right)}$, and the time derivatives of the carrier densities are set to zero. We find two LP solutions: $\varphi=0$ along the $x$-axis and $\varphi=\pi / 2$ along the orthogonal direction. Since we observed that our results are only weakly dependent on $\varepsilon_{a}$, we take for simplicity $\varepsilon_{a}=0$ in the rest of the paper. In this limit, $\omega_{ \pm}=-\varepsilon_{p}$ for a $x$-LP solution while $\omega_{ \pm}=\varepsilon_{p}$ for a $y$-LP solution. The total carrier inversions are in turn given by

$\bar{D}_{1,2}=\frac{\mu_{1,2}\left(1+a_{2,1} A\right)+\mu_{2,1} c_{12,21}}{(1+A)\left(1+a_{2} A\right)-c_{12} c_{21}}$.

The difference of the carrier inversion $\bar{d}_{1,2}=0$ in LP states. The total intensity, $A=2 Q^{2}$, satisfies the gain clamping condition $\bar{D}_{1} \pm \bar{d}_{1}+\bar{D}_{2} \pm \bar{d}_{2}=1$, leading to a quadratic equation that reads

$a_{2} A^{2}+\left[a_{2}\left(1-\mu_{2}\right)+\left(1-\mu_{2}\right)\right] A+1-\mu_{1}\left(1+c_{21}\right)-\mu_{2}\left(1+c_{12}\right)-c_{12} c_{21}=0$.

For our choice of parameters, Eq. (5) has only one positive root. From a linear stability analysis $^{9}$ of the two LP solutions, the linearized system (1)-(3) decouples into two subsets, one $(4 \times 4)$ that accounts for the stability of the total intensity, and one $(4 \times 4)$ that describes the polarization stability. Due to the arbitrariness in a global phase ${ }^{10}$, the actual dimension of the total intensity subset is three. As the carrier decay rates $\gamma_{1}$ and $\gamma_{2}$ are typically $o\left(10^{-3}\right)$, a simplified analysis can be performed. Applying the Routh-Hurwitz criteria ${ }^{11}$, and neglecting $o\left(\gamma_{1} \gamma_{2}\right)$, we obtain the boundary stability conditions ${ }^{12}$ 
$\gamma_{1}^{2} \mu_{1}+\gamma_{2}^{2} a_{2} \mu_{2}+\left(a_{2} \gamma_{2}-\gamma_{1}\right)\left[\left(\gamma_{2} c_{21}+\gamma_{1} c_{12}\right) \bar{D}_{1}-\gamma_{1} c_{12}\right]=0$,

$\Omega^{2}=\frac{1}{2}\left[\gamma_{1} \bar{D}_{1}+a_{2} \gamma_{2} \bar{D}_{2}\right] A>0$.

When Eq. (6) is fulfilled, the total intensity loses its stability through a Hopf bifurcation, giving rise to pulsations at frequency $\Omega$. As already mentioned, the dimension of the polarization subset is four and an analytical approach is quite involved. Alternatively, we numerically find the conditions for the stability boundaries of the polarization in a phase diagram $\mu_{1} / \mu_{t h}$ vs the birefringence $\gamma_{p} \equiv \varepsilon_{p} \kappa$, where $\mu_{t h}$ is the laser threshold. Four different regions can be distinguished in Fig. 1: in region A only the $y$-LP solution is stable, while in region B we observe total intensity pulsations in a stable linear polarization. In this case, the time traces are similar to those of SPs in EELs, disregarding polarization degrees of freedom. The most interesting regimes are region $\mathrm{C}$ with coupled pulsations of polarization and total intensity, and D where polarization pulsations with almost constant total intensity appear. In the region $\mathrm{C}$ both total intensity and polarization are unstable. The time traces of the two polarization components [See Fig. 2(A)] show amplitude modulation (AM) with the shape of strong pulses. We have also analyzed the modification of the stability diagram, shown in Fig. 1, against variations of the device and physical parameters. In particular, we find that the numerical line delimiting the regions $\mathrm{B}$ and $\mathrm{C}$ takes place for larger birefringence values when increasing the spin flip rate. In order to better characterize the dynamics in regions $\mathrm{C}$ and $\mathrm{D}$, we compute the power spectra of the total intensity $\left(\operatorname{PS}(\omega) \equiv\left\langle|\tilde{P}(\omega)|^{2}\right\rangle\right)$ and the optical spectra $\left(\operatorname{OS}_{x, y}(\omega) \equiv\left\langle\left|\tilde{F}_{x, y}(\omega)\right|^{2}\right\rangle\right)$ of both linear components, with $P(t) \equiv\left|F_{x}\right|^{2}+\left|F_{y}\right|^{2}$. Since the effect of spontaneous emission noise has been demonstrated to be important in self-pulsating lasers ${ }^{1,13}$, we add Langevin noise sources to the field equations ${ }^{14}$. The power spectrum [Fig. 2(B)] shows a fundamental frequency (corresponding to Eq. (7) near the bifurcation) and several harmonics. In addition to the AM, the two linear components undergo strong phase modulation (PM). The polarization resolved optical spectra [Fig. 2(C)] show, in both polarizations, an asymmetric double-peaked profile typical of a gain switched semiconductor laser ${ }^{15}$. The ratio between the width of the total 
optical spectrum [Fig. 2(C)], which represents the averaged phase-sweep during each pulse, and the fundamental peak position, is $\sim 40$ being of the same order of the experimentally reported value ${ }^{6}$. When continuously increasing the current level $\mu_{1}$ (at constant $\gamma_{p}$ ) from region $\mathrm{C}$ to $\mathrm{D}$, the fundamental frequency increases until the locus Eq. (6) is crossed, giving rise to a regime where the total intensity is stable while polarization is unstable. We observe that this transition is not sharp, but the optical pulses smoothly reshape into a chaotic waveform when crossing the horizontal line in Fig. 1. Increasing further the injection current, well inside region $\mathrm{D}$, these chaotic forms lead to a regular oscillation. In the later, the time traces [Fig. 3(A)] show a nearly out-of-phase AM composed by harmonic pulsations in the two polarization components. Consequently, the power spectrum [Fig. 3(B)] shows a single peak at this frequency. A harmonic PM is also present, in both polarizations, as shown in the polarization resolved OS [Fig. 3(C)]. In the experiments ${ }^{6}$, a similar transition from strongly chirped pulses to harmonic pulsations was also found when increasing the pump. Our results in region $\mathrm{C}$ and $\mathrm{D}$ are then in agreement with the experimentally reported power and optical spectra. The behavior observed in region $\mathrm{C}$ suggests that the phase dynamics is dominated by the phase-amplitude coupling, while the total intensity shows the usual self-pulsations induced via saturable absorption as in EEL ${ }^{4}$. On the contrary, in region D the polarization instability leads to time dependent harmonic solutions at almost constant total intensity. This result can be regarded as a sign of mode partition among the two polarization components. Our conjecture is also supported by the optical spectrum that is arranged in multiple peaks, a clear sign of the out-of-phase dynamics of the polarization pulsations.

In conclusion, we have demonstrated that polarization self-pulsations in VCSELs are possible under certain operation conditions. As a result of analytical and numerical analysis of our model, we have found regions of stable operation, coupled pulsations of the polarization and the total intensity, and polarization self-pulsations at nearly constant total intensity. These regions that appear when changing the injection current compare successfully with reported experimental results. This fact suggests that our model is a suitable framework 
to study self-pulsations in vertical-cavity surface-emitting lasers in presence of polarization effects.

This work has been funded by the European Commission through the VISTA HP-TRN, Marie Curie IF MCFI-2000-00617, and the Spanish MCyT under projects TIC99-0645-C0502 and BFM2000-1108. 


\section{REFERENCES}

1. C. R. Mirasso et al, IEEE J. Quantum Electron. 35, 764 (1999); M.Yamada, IEEE J. Quantum Electron. 29, 1330 (1993); R. W. Dixon, W.B. Joyce, IEEE J. Quantum Electron. 15, 470 (1979).

2. P. Rees et al, IEEE J. Quantum Electron. 35, 221 (1999).

3. C. Juang et al, IEEE J. Quantum Electron. 36, 300 (2000).

4. M. Yamada and T. Higashi, IEEE J. Quantum Electron. 27, 380 (1991).

5. K. J. Ebeling in Semiconductor Quantum Optoelectronics, edited by A. Miller, M.Ebrahimzadeh and D.M. Finlaynson (Institute of Physics, Bristol, 1999), p.339.

6. M. B. Willemsen et al, Appl. Phys. Lett. 77, 3514 (2000).

7. J. A. Hudgings et al, IEEE J. Quantum Electron. 5, 512 (1999).

8. T. W. Carr and T. Erneux, IEEE J. Quantum Electron. 37, 1171 (2001).

9. M. San Miguel et al, Phys. Rev. A 52, 1728 (1995).

10. J. M. Regalado et al, IEEE J. Quantum Electron. 33, 765 (1997).

11. G. A. Korn and T. M. Korn, Mathematical Handbook for Scientist and Engineers, Mc Graw-Hill, NY (1968).

12. A. Scirè, J. Mulet, C. R. Mirasso, and M. San Miguel. To be submitted in SPIE Procs. (2002).

13. G. H. M. van Tartwijk and M. San Miguel, IEEE J. Quantum Electron. 32, 1191 (1996).

14. J. Mulet, C. R. Mirasso and M. San Miguel, Phys. Rev. A 64, 023817 (2001).

15. See for instance G. P. Agrawal and N. K. Dutta, Long wavelength semiconductor lasers, Van Nostrand, NY (1986), p. 265. 
*Permanent Address: Departament de Física, Universitat de les Illes Balears. E-07071 Palma de Mallorca, Spain. 


\section{FIGURES}

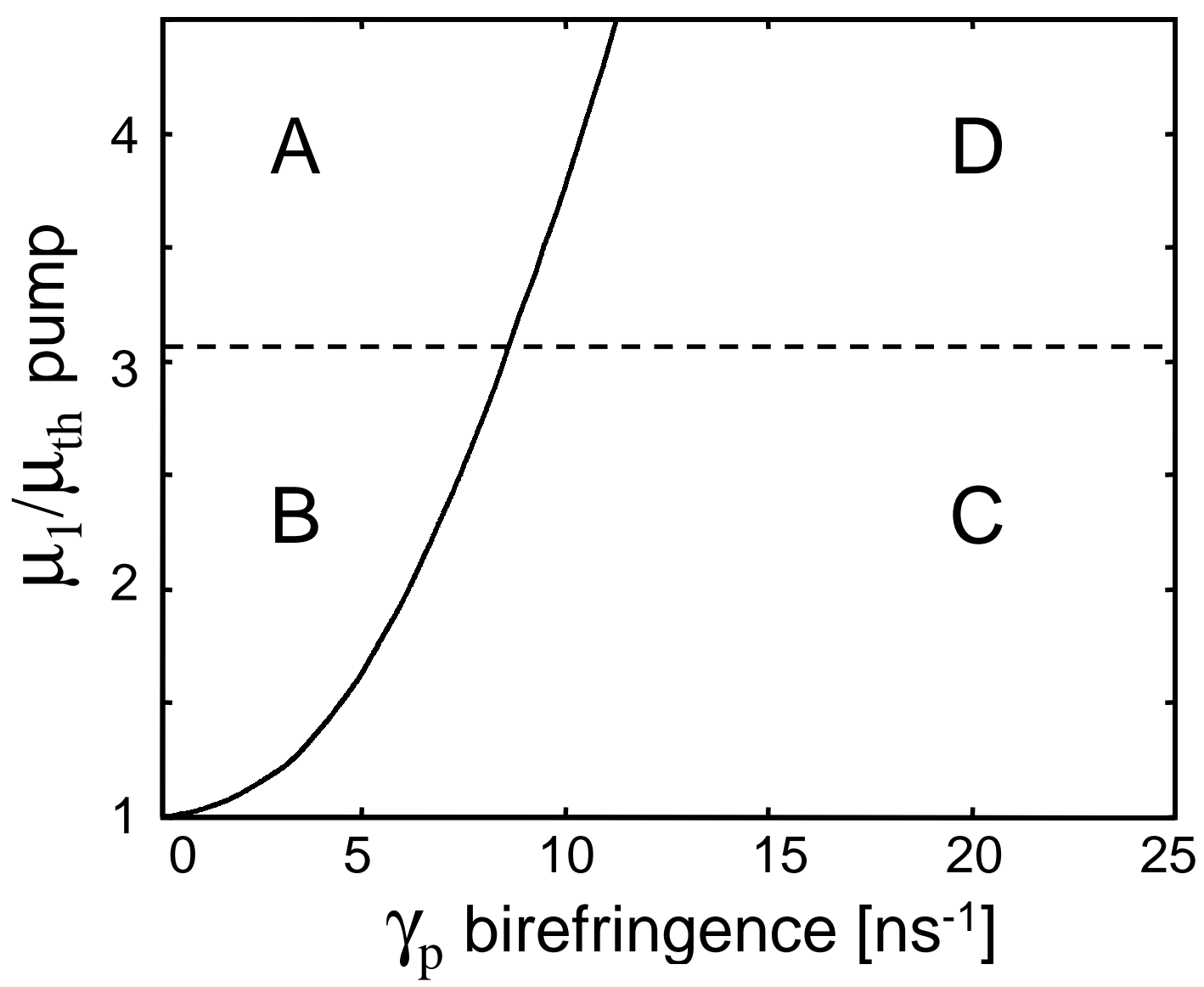

Fig. 1. Phase diagram $\left(\mu_{1} / \mu_{t h}\right.$ vs $\left.\gamma_{p}=\varepsilon_{p} \kappa\right)$ for the stability of the linearly polarized solutions. The four regions are A) $y$-LP stable, B) pulsations in one polarization, C) coupled total intensity and polarization pulsations and D) polarization pulsations. The parameters are: $\mu_{2}=-32$, $\gamma_{1}=1.09 \times 10^{-3}, \gamma_{2}=1.13 \times 10^{-3}, \alpha=3, c_{12}=2.84 \times 10^{-2}, c_{21}=1.91, \gamma_{s 1}=\gamma_{s 2}=0.25$, $a_{2}=8.7$, and $\kappa=390 \mathrm{~ns}^{-1}$. 

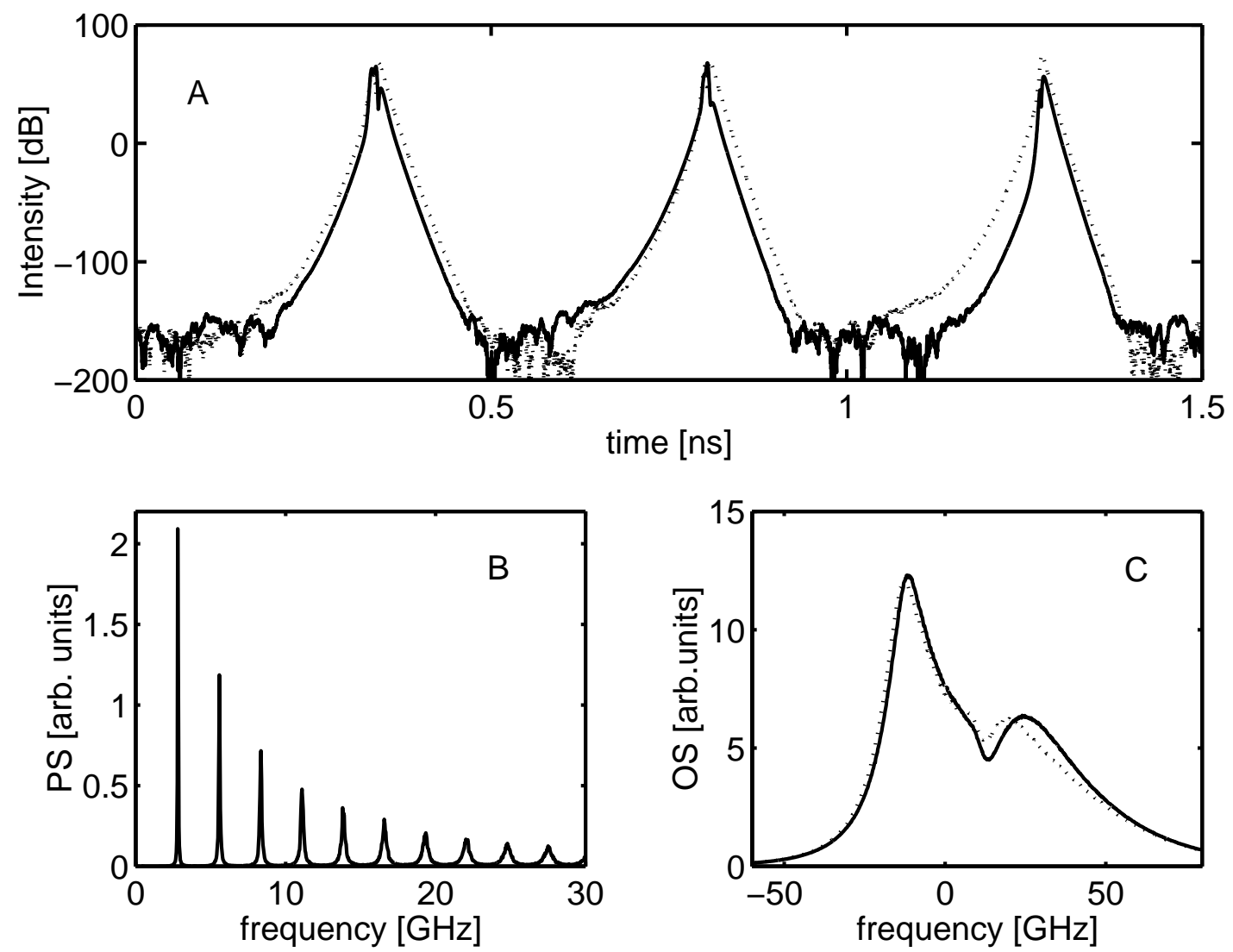

Fig. 2. A) time traces $x$-LP (dotted), $y$-LP (solid) in region $\mathrm{C}$ of figure $1,\left(\mu_{1} / \mu_{t h}=2, \gamma_{p}=20\right.$ $\mathrm{ns}^{-1}$ ), B) power spectrum of the total intensity, C) polarization resolved optical spectra for $x$-LP (dotted) and $y$-LP (solid). 

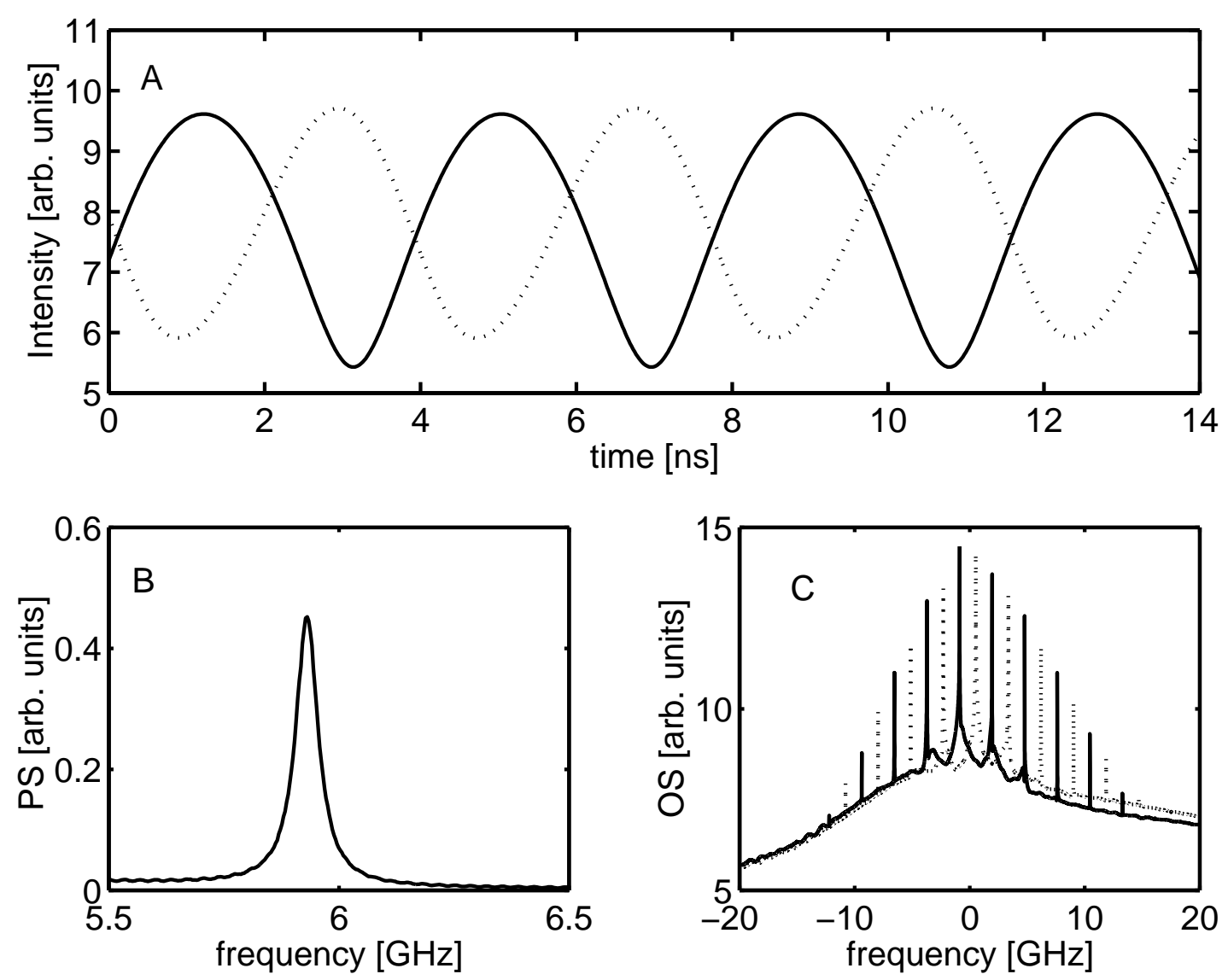

Fig. 3. A) time traces $x$-LP dotted, $y$-LP solid in region D of figure $1,\left(\mu_{1} / \mu_{t h}=4, \gamma_{p}=20\right.$ $\mathrm{ns}^{-1}$ ), B) total intensity power spectrum, C) polarization resolved optical spectra for $x$-LP (dotted) and $y$-LP (solid). Note that the arbitrary units are different with respect to Fig. 2. 